\title{
Résistance du Mollusque Prosobranche Potamopyrgus jenkinsi (E.A. Smith, 1899) aux températures décroissantes : étude expérimentale
}

c. Vareille-Morelt

Mots clés : Mollusques, Prosobranches, Potamopyrgus jenkirtsi, température, résistance thermique.

Chez Potamopyrgus jenkinsi soumis à des températures décroissantes, on note que :

(1) Les juvéniles sont légèrement moins résistants que les adultes.

(2) Les généra tions d'hiver (oligo ou eucalciques) placées dans leur eau d'origine sont plus résistantes que les générations d'été.

(3) Les adultes oligocalciques sont plus résistants que les adultes eucalciques.

(4) L'adaptation des mollusques oligocalciques à une eau eucalcique est peu favorisante et parfois même défavorisante (jeunes d'hiver, par exemple).

(5) L'adaptation des mollusques eucalciques à une eau oligocalcique fragilise les individus vis-à-vis des températures décroissantes.

Resistance of the prosobranch mollusc Potamopyrgus jenkinsi (E.A. Smith, 1889) to decreasing temperatures : an experimental study.

Kevwords : Mollusc, Prosobranch, Potamopyrgus jenkinsi, temperature, thermal resistance.

When Potamopyrgus jenkinsi was subjected to decreasing temperatures, it was found that:

(1) The juveniles were slightly less resistant than the adults.

(2) The winter generations (oligo or eucalcic), when kept in water in which they were found, were more resistant than the summer generations.

(3) Oligocalcic adults were more resistant than eucalcic adults.

(4) The adaptation of oligocalcic molluscs to eucalcic water was negligibly assisted and was perhaps also hampered (win. ter young for example).

(5) The adaptation of eucalcic molluscs to oligocalcic water weakened the resistance of individuals towards decreasing temperatures.

\section{1. - Introduction}

Lors d'une précédente étude (Va reille-Morel 1985), nous avons établi les températures létales supérieures de Potamopyrgus jenkinsi et montré que cellesci ne pouvaient justifier l'arrêt de la progression de l'espèce vers l'amont de nos rivières, dans la zone

1. Equipe de Malacologie Appliquée. U.E.R des Sciences Exactes et Naturelles. 123, avenue Albert-Thomas, 87060 Limoges Cedex. de contact calcaire-cristallin entre les départements de la Dordogne et de la Haute-Vienne.

Le présent travail a pour but de compléter cette analyse en expérimentant l'effet sur Potamopyrgus jenkinsi des températures décroissantes.

\section{2. - Matériel et méthodes}

\subsection{Matériel animal}

L'expérimentation porte sur des lots d'animaux analogues à ceux décrits dans Vareille-Morel (1985). 
Rappelons pour mémoire que deux populations sont testées : l'une provenant d'une eau oligocalcique $\left(17 \mathrm{mg} / \mathrm{l} \mathrm{Ca}{ }^{+}\right)$, l'autre d'une eau eucalcique (107 mg/l $\left.\mathrm{Ca}^{++}\right)$; chacune de ces populations est testée dans son eau d'origine d'une part et dans l'eau d'origine contraire, à laquelle elle a été préalablement adaptée.

Il est tenu compte en outre des générations d'été et d'hiver el, pour chacune d'elles, des écophases (juvéniles et adultes).

\section{2. - Protocole expérimental}

Les températures expérimentées varient de $12^{\circ} \mathrm{C}$ à $1^{\circ} \mathrm{C}$. Elles sont obtenues grâce à un bain conju. guant les effets d'un thermostat et d'un cryomat SFCASI, l'expérience étant menée pendant 24 heures à partir de l'obtention de la température choisir. Les autres données du protocole expérimental (nombre d'animaux, nature des récipients...) sont identiques à celles des températures croissantes.

\section{3. - Résultats}

\section{1. - Population oligocalcique naturelle (Tableau I)}

Jeunes et adultes d'été présentent sensiblement la même résistance aux températures décroissantes. La mortalité débute cependant un peu plus tôt chez les jeunes $\left(11^{\circ} \mathrm{C}\right.$ au lieu de $\left.9^{\circ} \mathrm{C}\right)$ et elle est totale à $4^{\circ} \mathrm{C}$ au lieu de $3^{\circ} \mathrm{C}$ chez les adultes. Les délais de récupération sont également plus longs chez les jeunes que chez les adultes pour une même température (par exemple, respectivement 7,5 jours pour 4,5 jours à $6^{\circ} \mathrm{C}$ ).

En hiver, les adultes sont plus résistants que les jeunes (respectivement par exemple $34 \%$ de mortalité contre $75 \%$ à $6^{\circ} \mathrm{C}$ ). Par contre, les délais de récupération sont voisins dans les deux catégories. Les deux écophases hivernales résistent mieux que leurs congénères d'été. Chez juvéniles et adultes d'hiver, la mortalité totale est atteinte respectivement à $3^{\circ} \mathrm{C}$ et $2^{\circ} \mathrm{C}$ contre $4^{\circ} \mathrm{C}$ et $3^{\circ} \mathrm{C}$ pour les écophases d'été.

Tableau I : Populations oligocalciques placées dans leur eau d'origine.

\begin{tabular}{|c|c|c|c|c|c|c|c|c|}
\hline \multirow{3}{*}{$\begin{array}{l}\text { Lots } \\
\mathrm{T}^{\circ} \mathrm{C}\end{array}$} & \multicolumn{4}{|c|}{ Elé } & \multicolumn{4}{|c|}{ Hiver } \\
\hline & \multicolumn{2}{|c|}{ Jeunes } & \multicolumn{2}{|c|}{ Adultes } & \multicolumn{2}{|c|}{ Jeunes } & \multicolumn{2}{|c|}{ Adultes } \\
\hline & $\begin{array}{c}\text { Mortalité } \\
\text { en } \%\end{array}$ & $\begin{array}{c}\text { Délai } \\
\text { en jours }\end{array}$ & $\begin{array}{c}\text { Mortalité } \\
\text { en \% }\end{array}$ & $\begin{array}{c}\text { Délai } \\
\text { en jours }\end{array}$ & $\begin{array}{c}\text { Mortalité } \\
\text { en } \%\end{array}$ & $\begin{array}{c}\text { Délai } \\
\text { en jours }\end{array}$ & $\begin{array}{c}\text { Mortalite } \\
\text { en } \%\end{array}$ & $\begin{array}{l}\text { Délai } \\
\text { en jours }\end{array}$ \\
\hline 1 & 100 & - & 100 & - & 100 & - & 100 & - \\
\hline 2 & 100 & - & 100 & - & 100 & - & 100 & - \\
\hline 3 & 100 & - & 100 & - & 100 & $\rightarrow$ & 94 & 9 \\
\hline 4 & 100 & - & 99 & 9 & 99 & 9,5 & 86 & 8,5 \\
\hline 5 & 99 & 9,5 & 91 & 7,5 & 90 & 9 & 54 & 7,5 \\
\hline 6 & 75 & 7,5 & 80 & 4,5 & 75 & 4 & 34 & 6 \\
\hline 7 & 41 & 6,5 & 35 & 3,5 & 35 & 4 & 15 & 5 \\
\hline 8 & 25 & 5,5 & 29 & 3 & 5 & 2 & 14 & 2,5 \\
\hline 9 & 11 & 2 & 6 & 2,5 & 0 & 0,5 & 0 & 1,5 \\
\hline 10 & 5 & 1,5 & 0 & 0 & 0 & 0 & 0 & 0 \\
\hline 11 & 2 & 0 & 0 & 0 & 0 & 0 & 0 & 0 \\
\hline 12 & 0 & 0 & 0 & 0 & 0 & 0 & 0 & 0 \\
\hline
\end{tabular}


D'autre part, les délais de récupération sont sensiblement identiques pour les adultes. Par contre, les jeunes d'hiver récupèrent plus vite (sauf aux températures létales) que les jeunes d'été (par exemple 4 iours contre 7,5 jours à $5^{\circ} \mathrm{C}$ ).

\section{2. - Population eucalcique naturelle (Tableau II)}

En été, les adultes se révèlent légèrement plus récistants que les jeunes mais la température létale à $100 \%$ est cependant la même $\left(4^{\circ} \mathrm{C}\right)$ pour les deux écophases.

De même, la mortalité commence à s'observer dans les deux cas à $8^{\circ} \mathrm{C}$.

Les délais de récupération sont sensiblement identiaues et restent peu importants ( 5 jours à 5,5 jours) jusqu'aux températures prélétales extrêmes.

Les jeunes d'hiver sont moins résistants que les adultes d'hiver, mais, dans l'ensemble, les générations d'hiver sont nettement plus résistantes que celles d'été. La mortalité des deux écophases s'observe respectivement à $3^{\circ} \mathrm{C}$ et $2^{\circ} \mathrm{C}$ contre $4^{\circ} \mathrm{C}$ en été.
Les délais de récupération, plus longs chez les jeunes à partir de $6^{\circ} \mathrm{C}$ sont, dans l'ensemble, moins longs qu'en été sauf pour les températures prélétales extrêmes.

\section{3 - Population oligocalcique adaptée à l'eau eucalcique (Tableau III)}

En été, les juvéniles sont légèrement moins résistants que les adultes. On note par exemple $50 \%$ de mortalité contre $35 \%$ à $7^{\circ} \mathrm{C}$. La mortalité totale est atteinte à $4^{\circ} \mathrm{C}$ pour les jeunes contre $3^{\circ} \mathrm{C}$ pour les adultes.

Les délais de récupération sont également légèrement plus longs chez les jeunes $(8,5$ jours contre 5,5 jours à $6^{\circ} \mathrm{C}$ par exemple).

Par rapport aux populations oligocalciques naturelles d'été, l'adaptation à l'eau eucalcique ne semble pas avoir été très bénéfique ; en effet, si la mortalité apparaît pour une température légèrement plus basse au moins pour les juvéniles $\left(10^{\circ} \mathrm{C}\right.$ au lieu de $11^{\circ} \mathrm{C}$ ), les délais de récupération sont pratiquement

Tableau II : Populations eucalciques placées dans leur eau d'origine.

\begin{tabular}{|c|c|c|c|c|c|c|c|c|}
\hline \multirow{3}{*}{$\begin{array}{l}\text { Lots } \\
\mathrm{T}^{\circ} \mathrm{C}\end{array}$} & \multicolumn{4}{|c|}{ Eté } & \multicolumn{4}{|c|}{ Hiver } \\
\hline & \multicolumn{2}{|c|}{ Jeunes } & \multicolumn{2}{|c|}{ Adultes } & \multicolumn{2}{|c|}{ Jeunes } & \multicolumn{2}{|c|}{ Adultes } \\
\hline & $\begin{array}{c}\text { Mortalité } \\
\text { en } \%\end{array}$ & $\begin{array}{l}\text { Délai } \\
\text { en jours }\end{array}$ & $\begin{array}{c}\text { Mortalité } \\
\text { en } \%\end{array}$ & $\begin{array}{c}\text { Délai } \\
\text { en jours }\end{array}$ & $\begin{array}{l}\text { Mortalité } \\
\text { en } \%\end{array}$ & $\begin{array}{c}\text { Délai } \\
\text { en jours }\end{array}$ & $\begin{array}{l}\text { Mortalité } \\
\text { en } \%\end{array}$ & $\begin{array}{c}\text { Délai } \\
\text { en jours }\end{array}$ \\
\hline 1 & 100 & - & 100 & - & 100 & - & 100 & - \\
\hline 2 & 100 & - & 100 & - & 100 & - & 100 & - \\
\hline 3 & 100 & - & 100 & - & 100 & - & 99 & 7 \\
\hline 4 & 100 & - & 100 & - & 95 & 8,5 & 91 & 5 \\
\hline $\mathbf{5}$ & 96 & 5 & 89 & 5,5 & 91 & 6 & 50 & 3 \\
\hline 6 & 76 & 4 & 62 & 3,5 & 61 & 3,5 & 45 & 1,5 \\
\hline 7 & 56 & 3 & 29 & 2,5 & 36 & 1,5 & 32 & 1,5 \\
\hline 8 & 22 & 1 & 21 & 1.5 & 0 & 1 & 20 & 1 \\
\hline 9 & 0 & 0 & 0 & 1 & 0 & 0 & 0 & 0 \\
\hline 10 & 0 & 0 & 0 & 1 & 0 & 0 & 0 & 0 \\
\hline 11 & 0 & 0 & 0 & 0 & 0 & 0 & 0 & 0 \\
\hline 12 & 0 & 0 & 0 & 0 & 0 & 0 & 0 & 0 \\
\hline
\end{tabular}


Tableau III : Populations oligocalciques adaptées à une eau cucalcique.

\begin{tabular}{|c|c|c|c|c|c|c|c|c|}
\hline \multirow{3}{*}{$\begin{array}{l}\text { Lots } \\
\mathrm{T}^{\circ} \mathrm{C}\end{array}$} & \multicolumn{4}{|c|}{ Eté } & \multicolumn{4}{|c|}{ Hiver } \\
\hline & \multicolumn{2}{|c|}{ Jeunes } & \multicolumn{2}{|c|}{ Adultes } & \multicolumn{2}{|c|}{ Jeunes } & \multicolumn{2}{|c|}{ Adultes } \\
\hline & $\begin{array}{l}\text { Mortalité } \\
\text { en } \%\end{array}$ & $\begin{array}{c}\text { Délai } \\
\text { en jours }\end{array}$ & $\begin{array}{c}\text { Mortalité } \\
\text { en } \%\end{array}$ & $\begin{array}{c}\text { Délai } \\
\text { en jours }\end{array}$ & $\begin{array}{c}\text { Mortalité } \\
\text { en } \%\end{array}$ & $\begin{array}{c}\text { Délai } \\
\text { en jours }\end{array}$ & $\begin{array}{c}\text { Mortalité } \\
\text { en } \%\end{array}$ & $\begin{array}{c}\text { Délai } \\
\text { en jours }\end{array}$ \\
\hline 1 & 100 & - & 100 & - & 100 & - & 100 & - \\
\hline 2 & 100 & - & 100 & - & 100 & - & 99 & 7,5 \\
\hline 3 & 100 & - & 100 & - & 97 & 10,5 & 99 & 5 \\
\hline 4 & 100 & - & 99 & 15 & 92 & 8,5 & 85 & 4,5 \\
\hline 5 & 96 & 12 & 92 & 7 & 90 & 7,5 & 75 & 3 \\
\hline 6 & 82 & 8,5 & 76 & 5,5 & 77 & 7 & 42 & 2,5 \\
\hline 7 & 50 & 5 & 35 & 4 & 46 & 6,5 & 25 & 2 \\
\hline 8 & 30 & 3,5 & 26 & 3 & 24 & 3 & 1 & 0,5 \\
\hline 9 & 11 & $2 ; 5$ & 10 & 1 & 7 & 2,5 & 0 & 0,5 \\
\hline 10 & 2 & 0,5 & 1 & 0,5 & 2 & 2 & 0 & 0 \\
\hline 11 & 0 & 0,5 & 0 & 0 & 0 & 0,5 & 0 & 0 \\
\hline 12 & 0 & 0 & 0 & 0 & 0 & 0 & 0 & 0 \\
\hline
\end{tabular}

semblables voire légèrement supérieurs dans les températures prélétales extrêmes (ex : 12 jours chez les jeunes adaptés à $5^{\circ} \mathrm{C}$ contre 9,5 jours pour les jelınes oligocalciques naturels et 15 jours chez les adultes oligocalciques adaptés à l'eau eucalcique à $4^{\circ} \mathrm{C}$ contre 9 jours à la même température chez les adultes oligocalciques non adaptés).

En hiver, les adultes se révèlent nettement plus récistants que les jeunes ( $25 \%$ de mortalité contre $46 \%$ à $7^{\circ} \mathrm{C}$ par exemple). De même, leurs délais de récupération sont largement inférieurs. On note ainsi 2,5 jours contre 7 jours à $6^{\circ} \mathrm{C}$.

Iuvéniles et adultes sont plus résistants à l'abaissement de température que leurs congénères d'été. La mortalité n'apparaît qu'à $8^{\circ} \mathrm{C}$ chez les adultes d'hiver contre $10^{\circ} \mathrm{C}$ chez les adultes d'été. Dans ces mêmes catégories, elle devient totale respectivement à $1^{\circ} \mathrm{C}$ et $3^{\circ} \mathrm{C}$.

De même, chez les juvéniles d'hiver, la mortalité est totale à $2^{\circ} \mathrm{C}$ contre $4^{\circ} \mathrm{C}$ en été.

Les délais de récupération sont peu modifiés entre juvéniles d'hiver et juvéniles d'été, mais, par contre, bien diminués chez les adultes d'hiver par rapport aux adultes d'été.
La résistance à l'abaissement thermique des deux écophases d'hiver adaptées à l'eau eucalcique est légèrement accrue par rapport aux deux écophases d'hiver non adaptées, au moins pour ce qui concerne la température létale à $100 \%$.

Pour les températures non létales, l'effet du calcium n'est pas réellement favorisant. On note même un début de mortalité chez les jeunes adaptés à $10^{\circ} \mathrm{C}$ au lieu de $8^{\circ} \mathrm{C}$ chez les jeunes d'hiver non adaptés.

Les délais de récupération sont améliorés seulement chez les adultes d'hiver par l'adaptation au milieu eucalcique. Ils sont plutôt aggravés par $l^{\prime}$ adaptation chez les juvéniles $\{6,5$ jours contre 4 jours à $7^{\circ} \mathrm{C}$, par exemple).

\section{4. - Population eucalcique adaptée à l'eau oligo- calcique (Tableau IV)}

Les générations d'été juvéniles ou adultes se comportent pratiquement de la manière vis-à-vis des températures décroissantes. De même, le délai de récupération est peu différent entre les deux écophases. La mortalité débute à $10^{\circ} \mathrm{C}$ alors qu'un 
Tableau IV : Populations eucalciques adaptées à une eau oligocalcique.

\begin{tabular}{|c|c|c|c|c|c|c|c|c|}
\hline \multirow{3}{*}{$\begin{array}{l}\text { Lots } \\
\mathrm{T}^{\circ} \mathrm{C}\end{array}$} & \multicolumn{4}{|c|}{ Eté } & \multicolumn{4}{|c|}{ Hiver } \\
\hline & \multicolumn{2}{|c|}{ Jeunes } & \multicolumn{2}{|c|}{ Adultes } & \multicolumn{2}{|c|}{ Jeunes } & \multicolumn{2}{|c|}{ Adultes } \\
\hline & $\begin{array}{c}\text { Mortalité } \\
\text { en } \%\end{array}$ & $\begin{array}{c}\text { Délai } \\
\text { en jours }\end{array}$ & $\begin{array}{c}\text { Mortalité } \\
\text { en } \%\end{array}$ & $\begin{array}{c}\text { Délai } \\
\text { en jours }\end{array}$ & $\begin{array}{c}\text { Mortalité } \\
\text { en \% }\end{array}$ & $\begin{array}{c}\text { Délai } \\
\text { en jours }\end{array}$ & $\begin{array}{l}\text { Mortalité } \\
\text { en } \%\end{array}$ & $\begin{array}{c}\text { Délai } \\
\text { en jours }\end{array}$ \\
\hline 1 & 100 & - & 100 & - & 100 & - & 100 & - \\
\hline 2 & 100 & - & 100 & - & 100 & - & 100 & - \\
\hline 3 & 100 & - & 100 & - & 100 & - & 98 & 15 \\
\hline 4 & 108 & - & 100 & - & 90 & 7,5 & 97 & 13 \\
\hline 5 & 99 & 6 & 94 & 8,5 & 77 & 4 & 88 & 8 \\
\hline 6 & 90 & 5,5 & 89 & 8,5 & 47 & 3,5 & 60 & 7,5 \\
\hline 7 & 84 & 5 & 79 & 5 & 12 & 2,5 & 38 & 4,5 \\
\hline 8 & 40 & 3 & 41 & 3,5 & 11 & 1 & 15 & 2,5 \\
\hline 9 & 15 & 2 & 13 & 2 & 1 & 1 & 4 & 1 \\
\hline 10 & 11 & 1,5 & 1 & 1,5 & 0 & 0 & 1 & 0.5 \\
\hline 11 & o & 1 & 0 & 0.5 & 0 & 0 & 0 & 0 \\
\hline 12 & 0 & 0 & 0 & 0 & 0 & 0 & 0 & 0 \\
\hline
\end{tabular}

faible délai de récupération est déjà nécessaire à $11^{\circ} \mathrm{C}$. La mortalité est totale dans les deux cas à $4^{\circ} \mathrm{C}$.

Pour les générations d'hiver, l'adaptation au milieu oligocalcique ne semble pas avoir une action favorisante sur les adultes par rapport aux juvéniles. On note ainsi respectivement $38 \%$ de morts contre $12 \%$ à $7^{\circ} \mathrm{C}$ et $60 \%$ contre $47 \%$ à $6^{\circ} \mathrm{C}$.

Les délais de récupération sont également sensiblement doublés chez les adultes par rapport aux juvéniles (par exemple 8 jours contre 4 jours à $5^{\circ} \mathrm{C}$ ).

La mortalité débute plus tôt chez les adultes $\left(10^{\circ} \mathrm{C}\right.$ au lieu de $9^{\circ} \mathrm{C}$ chez les juvéniles). Par contre, à $3^{\circ} \mathrm{C}$, il reste $2 \%$ de vivants chez les adultes contre $0 \%$ chez les juvéniles.

Les deux écophases d'hiver sont nettement plus récistantes que celles d'été placées dans les mêmes conditions. On observe par exemple $12 \%$ de mortalité chez les jeunes d'hiver contre $84 \%$ chez les jeines d'été à $7^{\circ} \mathrm{C}$. De même, $38 \%$ chez les adultes d'hiver contre $79 \%$ chez les adultes d'été.

Si on compare les résultats du Tableau IV avec ceux du Tableau II (populations eucalciques placées dans leur eau naturelle), on constate que, globalement, l'adaptation à l'eau oligocalcique a plutôt un effet défavorisant. La mortalité y est plus précoce $\left(10^{\circ} \mathrm{C}\right.$ en été et en hiver contre $8^{\circ} \mathrm{C}$ chez les populations naturelles).

Par rapport au Tableau I, les populations eucalciques adaptées à l'eau oligocalcique sont moins résistantes que les populations oligocalciques dans leur eau d'origine.

De même, par comparaison avec le Tableau III, il apparaît que les populations eucalciques adaptées à l'eau oligocalcique sont moins résistantes que les populations oligocalciques adaptées à l'eau eucalcique.

\section{4. - Discussion}

L'ensemble des résultats peut se résumer de la façon suivante :

- Les mollusques adultes eucalciques d'hiver placés dans leur eau d'origine sont plus résistants que ceux d'été.

- Les adultes oligocalciques d'hiver placés dans leur eau d'origine présentent des résultats comparables aux précédents. Ils semblent cependant plus résistants que les eucalciques. 
- L'adaptation des mollusques oligocalciques à une eau eucalcique n'a pas d'influence notablement favorisante à la résistance aux températures décroissantes comme nous l'avions constaté pour les températures croissantes (Vareille-Morel 1985). Parfois même, elle semble défavorisante (jeunes d'hiver par exemple où la mortalité débute à $10^{\circ} \mathrm{C}$ au lieu de $8^{\circ} \mathrm{C}$ ).

- L'adaptation des mollusques eucalciques à une eau oligocalcique fragilise également les individus vis-à-vis des températures décroissantes.

- Il y a une résistance légèrement supérieure chez les adultes que chez les juvéniles dans tous les cas de figure mais elle est bien moins nette que celle observée dans les expériences de résistance aux températures croissantes.

Nos résultats confirment ceux d'autres auteurs y compris sur d'autres espèces. Ainsi Vincent (1971) trouve que les Gammares de milieu oligocalcique sont plus résistants au froid que les Echinogammares de milieu eucalcique. Il note aussi que la résistance des formes eucalciques diminue dans le milieu hyoocalcique. Il est vrai que cet auteur expérimente sur des espèces différentes d'Amphipodes épigés mais peut-être ne faudrait-il pas exclure totalement l'hvpothèse d'espèces également différentes au moins du point de vue génétique en ce qui concerne Potamopyrgus jenkinsi mais cette hypothèse reste à vérifier.

Sweeney et Hastings (1961) donnent une intéressante synthèse de l'effet de la température sur les rythmes diurnes. A cette occasion, ils montrent sur plısieurs espèces l'effet (généralement ralentissant) des températures basses sur certains phénomènes physiologiques tels que la ponte ou l'activité nutritionnelle et sur le métabolisme en général.

Vernberg (1967) s'intéresse également au métabolisme de Mollusques parasités ou non par des Trématodes et note l'effet des températures basses ou élevées sur ces deux cas de figures.

Wolda (1964) met en évidence l'effet défavorable des températures décroissantes sur la ponte de Cevaea nemoralis et suggère $\mathrm{l}^{\prime}$ importance du lieu et de la date de récolte ainsi que des possibles différences génétiques.

Wood (1978) étudie les possibilités d'adaptation aux températures croissantes et décroissantes et confirme leur existence. Nous n'avons pas pour notre part abordé la question sous l'angle de l'acclimatation. Cependant, la résistance relativement plus grande des adultes d'hiver oligocalciques habitués à des eaux plus froides que les eucalciques, conforterait cette hypothèse.

\section{5. - Conclusion}

Dans notre précédente note (Vareille-Morel 1985), nous nous posions la question de savoir si les températures hivernales, basses en Limousin $\left(6^{\circ} \mathrm{C}\right.$ en moyenne avec une variation diurne de $2^{\circ} \mathrm{C}$ à $8^{\circ} \mathrm{C}$ ) pouvaient, en conjonction avec d'autres facteurs (qualité de l'eau, substrat, vitesse de courant...) constituer un frein voire un arrêt à la progession de Potamopyrgus jenkinsi dans nos eaux (Vareille-Morel 1985). Nous avons constaté, par ailleurs, que les températures décroissantes (conformément aux observations des auteurs signalés précédemment) ralentissaient le métabolisme. Les présents résultats indiquent qu'à $6^{\circ} \mathrm{C}$, les populations oligocalciques naturelles présentent un taux important de mortalité (75\%) chez les juvéniles. Joint aux autres ralentissements physiologiques (capacité nutritionnelle, fécondité, activité...) cet élément peut justifier la non-implantation de l'espèce dans notre région.

\section{Travaux cités}

Sweeney (B.M.) \& Hastings \{J.W.\}. 1961. - Effects of temperature upon diurnal rhythms. Cold. Spr. Harb. Symp. Quant. Biol. $1960,25: 87 \cdot 104$.

Vareille-Morel (C.). 1981. - Contribution à l'étude de Potantopyr gus jenkinsi (E.A. Smith, 1889). I. Biotopes et progression actuelle du Prosobranche dans les bassins supérieurs de la Dronthe et de sort affluent I'Tsle. Ann. Stat. Biol. Besse-enChandesse, 15: 166-220.

Vareille-Morel (C.) 1985. - Résistance du mollusque Prosobran. che Polamopyrgus jenkinsi (E.A. Smith, 1889) aux temperatu res croissantes : etude experimentale. Anmls Limnol, 21 (1): 19.24.

Vemberg (W.B.) \& Vernberg (F.J.) 1967. - Interrelationships between parasites and their hosts. III. Effect of larval Trematodes on the thermal metabolic response of their Molluscan host. Experimental Parasitology, 20:225-231.

Vincent (M.). 1971. - Résistance thermique et teneur en sels des eaux chez les Gammares épigés du Centre-Ouest. C.R. Soc. Biol., $165: 648$.

Wolda (H.) 1967 . - The effect of temperature on reproduction in some morphs of the landsnail Cepaea nemoralis (L.). Evolution, $21: 117-129$.

Wood (D.H.) 1978. - Temperature adaptation in the freshwater snail, Helisoma trivolvis (Say), in an artificially heated reservoir in the southeastern United States. J. Therm. Biol., 3 (4): 187-194. 